\title{
A microcantilever mechanical antenna
}

Cite as: Appl. Phys. Lett. 115, 083902 (2019); https://doi.org/10.1063/1.5109353

Submitted: 08 May 2019 . Accepted: 24 July 2019 . Published Online: 20 August 2019

G. Abadal (D) P. Bramon, M. López-Suárez, J. Agustí, and F. Torres

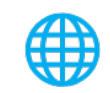

View Online

\section{ARTICLES YOU MAY BE INTERESTED IN}

A solid-state wind-energy transformer

Applied Physics Letters 115, 083901 (2019); https://doi.org/10.1063/1.5109776

Capacitive silicon micro-electromechanical resonator for enhanced photoacoustic spectroscopy

Applied Physics Letters 115, 081106 (2019); https://doi.org/10.1063/1.5098140

Acoustic metamaterial antennas for combined highly directive-sensitive detection

Applied Physics Letters 115, 053501 (2019); https://doi.org/10.1063/1.5107464

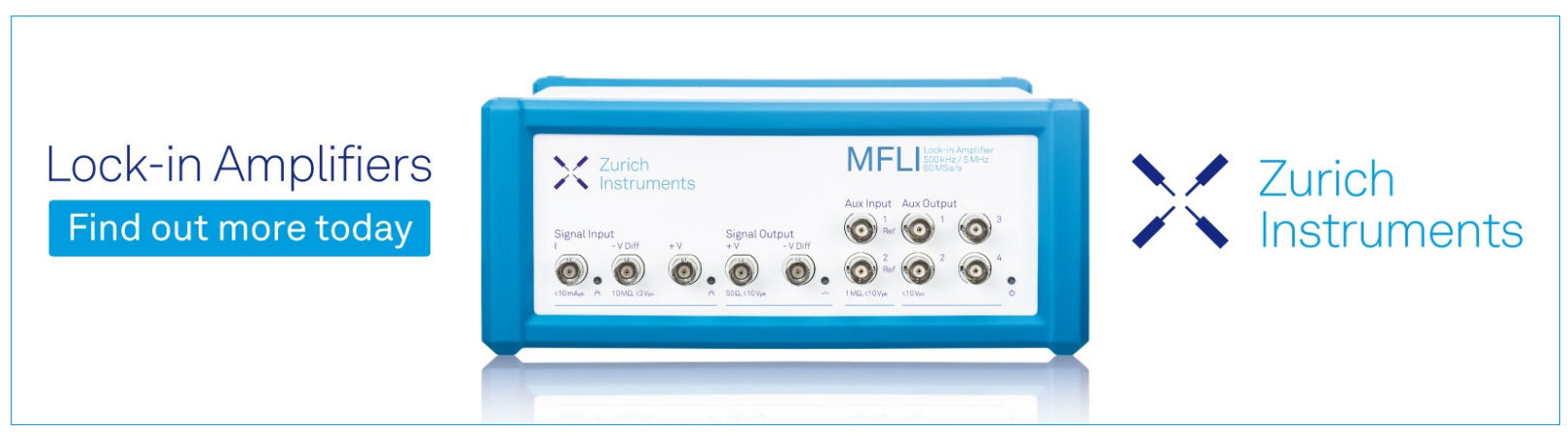




\title{
A microcantilever mechanical antenna
}

\author{
Cite as: Appl. Phys. Lett. 115, 083902 (2019); doi: 10.1063/1.5109353 \\ Submitted: 8 May 2019 - Accepted: 24 July 2019 . \\ Published Online: 20 August 2019

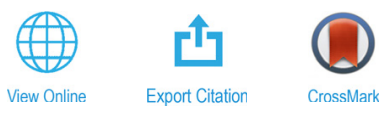

\author{
G. Abadal, ${ }^{1, a)}$ (D) P. Bramon, ${ }^{1, b)}$ M. López-Suárez, ${ }^{2}$ J. Agustí, $^{1, c)}$ and F. Torres ${ }^{\top}$
}

\author{
AFFILIATIONS \\ 'Departament d'Enginyeria Electrònica, Universitat Autònoma de Barcelona, Bellaterra 08193, Spain \\ ${ }^{2}$ Institut de Ciència de Materiales de Barcelona (ICMAB-CSIC), Campus de Bellaterra, 08193 Bellaterra, Barcelona, Spain \\ a) gabriel.abadal@uab.cat \\ b) Present address: Passatge Haití 7, 17800 Olot, Girona, Spain. \\ ${ }^{c)}$ Present address: Lear Corporation Engineering SL, C. Fusters 54, 43800 Valls, Tarragona, Spain.
}

\begin{abstract}
A mechanical antenna based on the interaction of an oscillating electric field with a permanent electric charge implanted in the free end of a clamped-free beam is proposed as a miniaturized alternative to conventional electromagnetic antennas. A prototype based on a silicon nitride triangular microcantilever has been fabricated, and its response to an oscillating electric field generated by a dipole antenna has been tested. The responsivity, defined as the vibration amplitude per electric field intensity unit, has been experimentally found to be $\Re=0.29 \mathrm{~nm} / \mathrm{kV} \mathrm{m}$. A figure of merit (FOM) to evaluate the power conversion efficiency per unit area of the microcantilever antenna has been defined and calculated to be $\mathrm{FOM}=0.216 \% \mathrm{~m}^{-2}$.
\end{abstract}

Published under license by AIP Publishing. https://doi.org/10.1063/1.5109353

A radio frequency rectenna, ${ }^{1}$ obtained by coupling an electromagnetic antenna with a current rectifier into a compact device, has been the key element for most of the energy harvesting ${ }^{2}$ and wireless power transfer devices ${ }^{3}$ that extract energy from electromagnetic radiation in the microwave bands. Optical rectennas, combining nanoantennas with ultrafast rectifiers, were proposed ${ }^{4}$ as an extrapolation of the radio frequency rectennas to enhance conversion efficiency of solar photovoltaic technologies over $80 \%{ }^{5,6}$ However, only a few experimental realizations have been reported until now, ${ }^{7,8}$ due to the lack of efficiency of actual electron devices in rectifying currents oscillating at hundreds of terahertz. On the other hand, the dimensions of a rectenna are mainly limited by the antenna component, which in a classical radioelectric configuration will always be a fraction of the radiation wavelength $(\lambda)$ and will be related to the radiation frequency ( $\mathrm{f}$ ) through $\lambda=\mathrm{c} / \mathrm{f}$ (with $\mathrm{c}$ being the speed of light). Thus, for microwave radiation ( $\mathrm{f}=3 \mathrm{GHz}$ to $\mathrm{f}=300 \mathrm{GHz}$ ), the antenna's characteristic length will range from $100 \mathrm{~mm}$ to $1 \mathrm{~mm}$ and for optical radiation from few hundreds of nanometers (visible) to few microns (infrared). ${ }^{9}$ Metamaterials have been used as a strategy to improve the performance of energy harvesting structures in different energy source domains such as solar radiation, ${ }^{10,11}$ acoustic/mechanical, ${ }^{1,13}$ radio frequency, ${ }^{14,15}$ and thermal. ${ }^{16}$ In a previous work, a mechanical antenna based on a resonating nanotube cantilever was proposed as an alternative to the classical radioelectric antenna that shrinks the dimensions of the radiation detector to the nanoscale. ${ }^{17}$ Such a reduction in dimensions is due to the fact that in a mechanical antenna, the characteristic length is related to the radiation frequency through the speed of sound, $\mathrm{v}=8.3 \times 10^{3} \mathrm{~m} \mathrm{~s}^{-1}$ (for silicon), instead of the speed of light as in a classical radioelectric antenna. The five orders of magnitude difference between both speed values is translated directly to a difference of the same order in the antenna dimensions. In such a mechanical antenna, the oscillating electric field associated with the propagating electromagnetic wave interacts with a charge density localized at the very end of the nanotube and, as a consequence, the mechanical resonance of the antenna is excited. In this case, the authors induced the charge by applying a high voltage between the nanotube and a closely placed electrode. ' So, consequently, such a configuration only makes sense if an active RF detector wants to be implemented since a voltage polarization is needed for the antenna operation. In this letter, we propose a passive mechanical antenna based on a microcantilever with a permanent charge implanted ${ }^{18}$ so that any bias voltage is needed for the antenna to work. Thus, for instance, RF energy harvesting and RF power transfer applications can be implemented using this antenna since any polarization voltage is needed for the transducer antenna operation. On the other hand, although the decay time of the implanted charge in silicon nitride passivation layers is on the order of months, ${ }^{19}$ a Teflon coating can be used to increase lifetime to hundreds of years. ${ }^{20}$ In order to demonstrate the performance of the mechanical antenna in reception, we generated an oscillating electric field from a conventional dipole antenna tuned to emit at the resonance frequency of the receiving mechanical antenna. The mechanical vibrations induced in the 
cantilever based antenna are measured by means of a laser optical setup and compared to theoretical predictions.

The mechanical antenna is fabricated from a commercially available silicon nitride triangular cantilever. ${ }^{21}$ Its geometry, as depicted in Fig. 1 (inset), is defined by its body thickness, $\mathrm{t}_{\mathrm{Si} 3 \mathrm{~N} 4}=600 \mathrm{~nm}$, length, $\mathrm{L}=200 \mu \mathrm{m}$, base width, $\mathrm{B}=184 \mu \mathrm{m}$, and arm width, $\mathrm{w}=28 \mu \mathrm{m}$. The cantilever is supplied with a $65 \mathrm{~nm}$ thick $\mathrm{Cr} / \mathrm{Au}$ layer coating the backside of the structure to enhance optical reflectivity. The datasheet typical values for resonance frequency and spring constant are $17 \mathrm{kHz}$ and $0.08 \mathrm{~N} / \mathrm{m}$, respectively. A permanent charge is implanted locally at the tip of the cantilever by means of a simple method reported in a previous work, ${ }^{18}$ which consists in applying $15 \mathrm{~V}$ for $30 \mathrm{~min}$ between the $\mathrm{Cr} / \mathrm{Au}$ coated side of the cantilever and a gold coated sample contacting its opposite uncoated side. Following this procedure, the mechanical antenna is charged with approximately ${ }^{10} \mathrm{q}=10 \mathrm{fC}$.

In order to excite the mechanical antenna, a conventional $1 \mathrm{~m}$ long dipole antenna emitter is designed and fabricated [see Fig. 1(a)]. For this characteristic length, $1=1 \mathrm{~m}$, and taking into account that the radiation wavelength is $\lambda=\mathrm{c} / \mathrm{f}=17.6 \times 10^{3} \mathrm{~m}$, the antenna corresponds to an infinitesimal dipole $(\lambda \gg 1){ }^{22}$ The dipole antenna is loaded with a variable LC impedance in order to allow a fine tuning of the emitter self-resonance around the mechanical resonance frequency of the microcantilever antenna. Although such an infinitesimal dipole antenna is not radiating efficiently at this frequency because the radiation wavelength is 3 orders of magnitude larger than the antenna length, it is enough for our purpose since the amplitude values of the oscillating electric field generated by the dipole are high enough to excite the mechanical antenna in the near-field region of the dipole $(\mathrm{kr} \ll 1)$. In these conditions, the magnitudes of the vertical $(\mathrm{z})$ and radial $(\rho)$ electric field components are given by ${ }^{22}$

$$
E_{z}=-j \eta \frac{I_{d}}{4 \pi}\left(\frac{1}{R_{1}}+\frac{1}{R_{2}}-\frac{2}{r}\right) \text {, }
$$

$$
E_{\rho}=j \eta \frac{I_{d}}{4 \pi} \frac{1}{\rho}\left(\frac{z-l / 2}{R_{1}}+\frac{z+l / 2}{R_{2}}-\frac{2 z}{r}\right),
$$

where $\eta=377 \Omega$ is the impedance of the propagating medium, $\mathrm{I}_{\mathrm{d}}$ is the magnitude of the current in the dipole, and $\mathrm{r}, \mathrm{R}_{1}, \mathrm{R}_{2}, \rho$, and $\mathrm{z}$ are the radial and cylindrical coordinates as defined in Fig. 1(a). As it can be seen, the radial and vertical components of the electric field are in time counterphase. On the other hand, as it is also depicted in Fig. $1(\mathrm{a})$, the microcantilever antenna is placed at a certain point defined by cylindrical coordinates $(\rho, \mathrm{z})$ and oriented with a certain tilt angle, $\theta$, with respect to the radial direction. Thus, the electric field in the direction normal to the cantilever free end surface, $E_{n}$, resulting from the projection of the electric field components $\mathrm{E}_{\rho}$ and $\mathrm{E}_{\mathrm{z}}$, excites the out-of-plane resonance mode of the cantilever (n-direction) when an oscillating force, $F_{n}=q \times E_{n}$, is developed in the same direction, as a consequence of the interaction of the electric field and the permanent charge, q. To measure the dynamic response of the microcantilever antenna, we use an optical readout system which is described in Fig. 1(b). A red HeNe laser beam $(632.8 \mathrm{~nm}, 15 \mathrm{~mW})$ is focused at the tip of the cantilever using a microscope objective (Mitutoyo M Plan APO SL20X NA $=0.28$ ) with the visual support of a CCD camera. The angle modulation of the reflected beam generated by the cantilever vibration is detected through a position sensitive detector (PSD, New Focus 2930). The electrical signal at the output of the PSD is analyzed by means of a network analyzer (Agilent 5100A). The cantilever is attached to a piezoelectric shaker in order to eventually produce a direct excitation of the out-of-plane vibration of the cantilever. The cantilever and piezoshaker set are mounted in an xyz micropositioner to accurately place the cantilever tip at the laser beam focus. The output of the network analyzer is connected to the dipole antenna when a measurement of the mechanical antenna response to the dipole generated electric field wants to be performed. Alternatively, the output of the network analyzer is connected to the piezoelectric shaker when a direct inertial excitation of the cantilever wants to be induced.

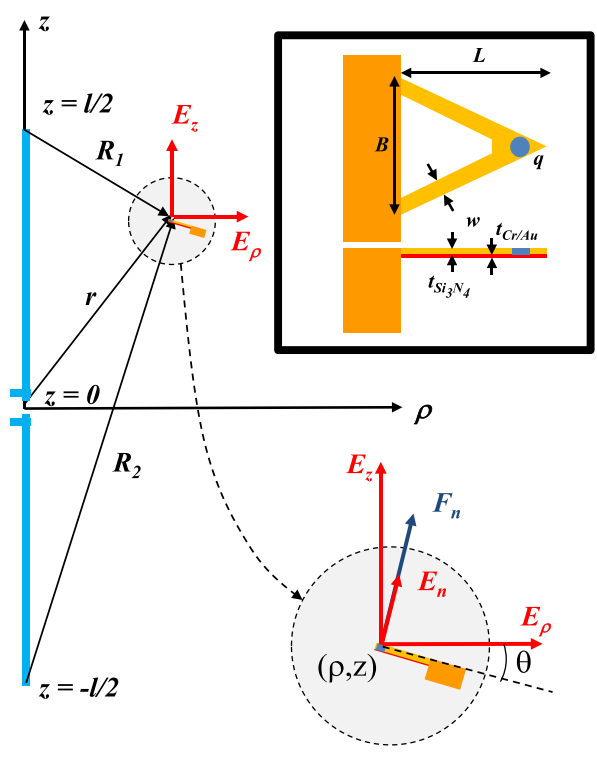

(a)

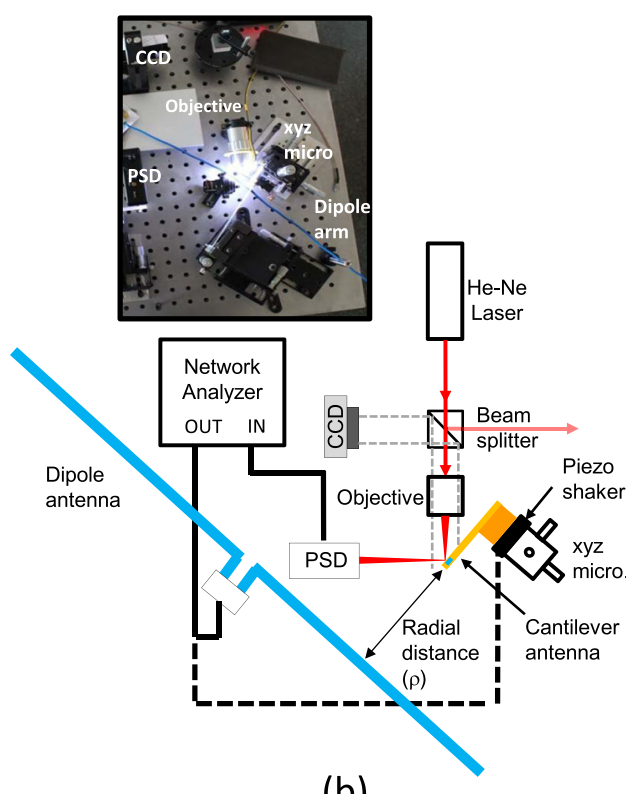

(b)
FIG. 1. (a) Scheme of the emitting $1 \mathrm{~m}$ long dipole antenna and the microcantilever mechanical antenna, placed at a generic point defined by cylindrical coordinates $(\rho, z)$ in the near-field region of the dipole. A tilt angle of the cantilever, $\theta$, is defined with respect to the radial axis. Inset: top and lateral schematic views of the microcantilever with an implanted charge. (b) Scheme and picture (inset) of the measurement setup. 
A first set of measurements is performed to demonstrate that the microcantilever antenna can be excited by the oscillating electric field generated by the dipole antenna. During all these first measurements, the microcantilever antenna is placed at $\mathrm{z}=1 / 4$ and $\rho=10 \mathrm{~mm}$ with a tilt angle $\theta=0^{\circ}$. Previously, the resonance frequency of the first outof-plane mode of the cantilever has been experimentally determined from the mechanical frequency response measured with the network analyzer and the piezoelectric shaker configuration (red circles, Fig. 2, Piezo Excited Cantilever, PEC, curve). The obtained resonance frequency and $\mathrm{Q}$-factor are $\mathrm{f}_{1}=11.4 \mathrm{kHz}$ and $\mathrm{Q}=20$, respectively. Additionally, the dipole antenna has been tuned to have its resonance at $f_{\text {res, } D}=11 \mathrm{kHz}$, close to the resonance of the cantilever, as it is evidenced by the measured frequency response of the dipole antenna current (filled blue triangles, in Fig. 2, Dipole Antenna, DA, curve).

Figure 2 also shows the frequency response of the cantilever outof-plane vibration measured through the optical setup when the piezoshaker is disconnected and the dipole antenna is emitting (filled black squares in Fig. 2, Dipole Excited Cantilever, DEC, curve). As it can be seen, the last frequency response of the microcantilever antenna results from the convolution between its pure mechanical response and the electrical response of the dipole antenna. To highlight this fact, the product of the DA and PEC curves is calculated and represented in Fig. 2 (green dashed-dotted curve). As it can be seen, a very good match between DEC and DAxPEC curves verifies our hypothesis. Figure 2 (open symbol curves) also shows the results of the same kind of experiment described above, but at different detuning conditions, i.e., the dipole antenna resonance has been moved from $\mathrm{f}_{\text {res }, \mathrm{D}}=11 \mathrm{kHz}$ $(-400 \mathrm{~Hz}$ detuning $)$ to $\mathrm{f}_{\text {res, } \mathrm{D}}=12 \mathrm{kHz}(+600 \mathrm{~Hz}$ detuning $)$. Note that the product of the dipole antenna and piezo excited cantilever curves (DAxPEC) matches with the dipole excited response of the microcantilever antenna (DEC).

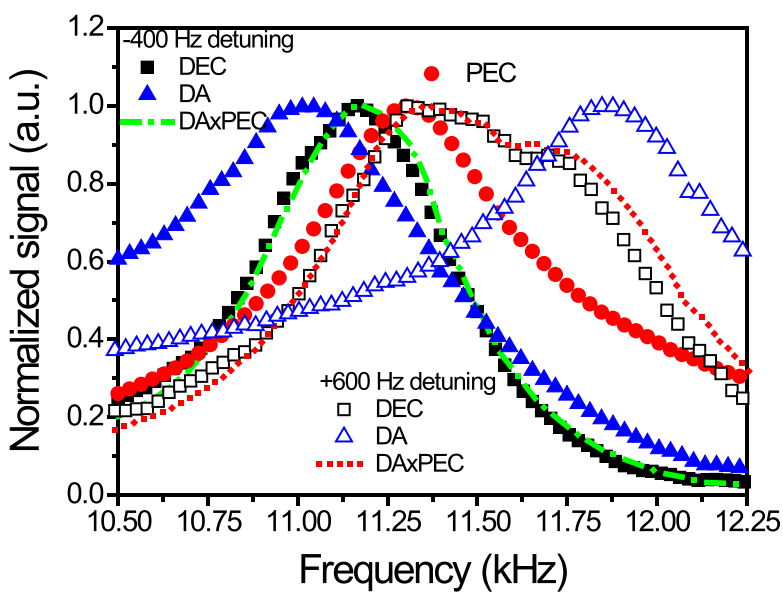

FIG. 2. Normalized frequency response of the cantilever out-of-plane vibration excited by the piezoelectric shaker (filled red circles, Piezoelectric Excited Cantilever, PEC) or by the electric field generated from the dipole antenna (black squares, Dipole Excited Cantilever, DEC), when the dipole is detuned $-400 \mathrm{~Hz}$ (filled symbols) and $+600 \mathrm{~Hz}$ (open symbols) from the resonance frequency of the microcantilever antenna. The dipole antenna frequency response (blue triangles, Dipole Antenna, DA) and its product by the PEC curve (dotted and dash-dotted line curves, DAxPEC) are also plotted in both detuning conditions.
In a second set of measurements, the dependence of the mechanical antenna response on the radial distance, $\rho$, from the dipole emitter has been tested. For this purpose, the dipole antenna has been excited with a $10 \mathrm{dBm}$ signal at $11.4 \mathrm{kHz}$, which means that no detuning is introduced and both antennas are perfectly matched. In these conditions, the above described DEC signal at the output of the PSD has been measured when the microcantilever antenna is placed at different radial distances and keeping constant the vertical position and tilt angle at $\mathrm{z}=1 / 4$ and $\theta=0^{\circ}$, respectively. The height of the resonance peak values in terms of the vibration amplitude detected at the PSD output are plotted as a function of the radial distance in Fig. 3. A fitting analysis of the experimental points to the theoretical curves derived from Eqs. (1) and (2) reveals that the $\mathrm{E}_{\rho}$ component should prevail over the $\mathrm{E}_{\mathrm{z}}$ component in the contribution to the normal electric field $\left(\mathrm{E}_{\mathrm{n}}\right)$, which is only consistent with a deviation of $12^{\circ}$ from the nominal $\theta=0^{\circ}$ tilt angle. Indeed, the spatial decay dependence of $\mathrm{E}_{\rho}$ and $\mathrm{E}_{\mathrm{z}}$ with the radial distance at $\mathrm{z}=1 / 4$ (inset of Fig. 3) shows that only a dominant $\mathrm{E}_{\rho}$ component is compatible with the decay dependence on the radial distance experimentally measured.

Finally, Fig. 4 (linked to the left vertical axis) shows the dependence of the mechanical antenna response on the alignment to the electric field. Thus, it can be noticed that a clear peak of $3.5 \mathrm{~nm}$ is detected (red dotted curve) when the mechanical antenna is oriented in the optimum direction $\left(\mathrm{z}=1 / 4, \theta=0^{\circ}\right)$. Instead, when the mechani$\mathrm{cal}$ antenna is oriented in the direction of a null electric field $(\mathrm{z}=0$, $\theta=90^{\circ}$ ), no resonant response is detected (black dashed curve) in spite of being placed closer in the radial direction $(\rho=2.5 \mathrm{~mm})$.

Figure 4 (blue solid curve) also shows that the vibration amplitude is $0.35 \mathrm{~nm}$ when the mechanical antenna is placed at a radial

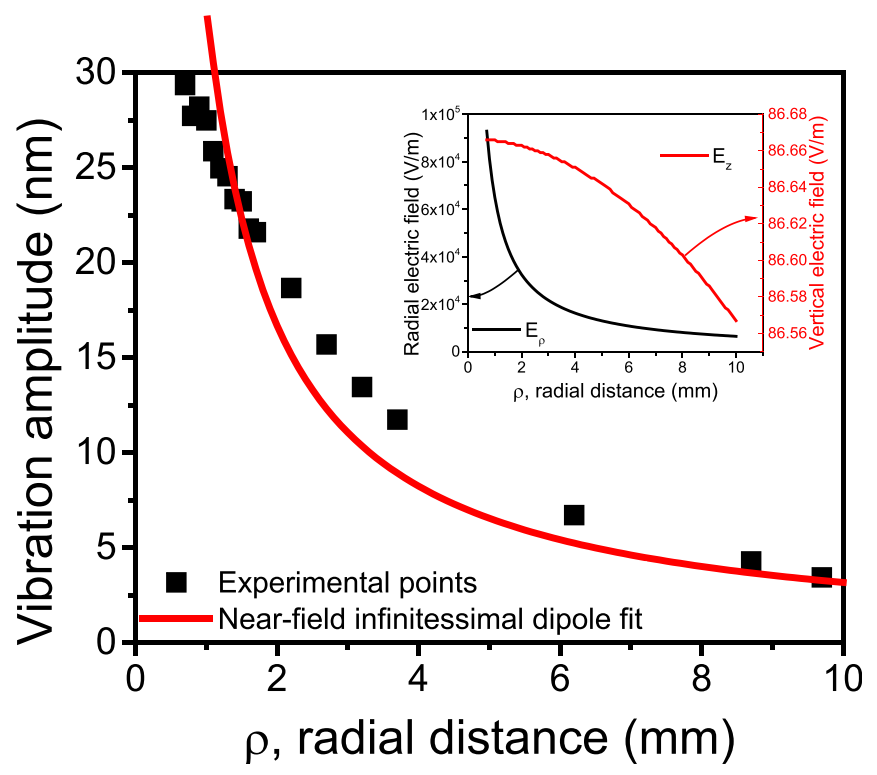

FIG. 3. Experimental and fitting decay curves in the radial direction, $\rho$, of the mechanical resonant peak when the microcantilever antenna is excited by the dipole emitter. Measurement conditions are $Z=1 / 4, \theta=12^{\circ}$. Inset: decay dependence of the two components of the electric field at $z=l / 4$ (radial, $E_{\rho}$, and vertical $E_{z}$ ) as described by the equations of the infinitesimal dipole in the near-field approximation [Eqs. (1) and (2)]. 


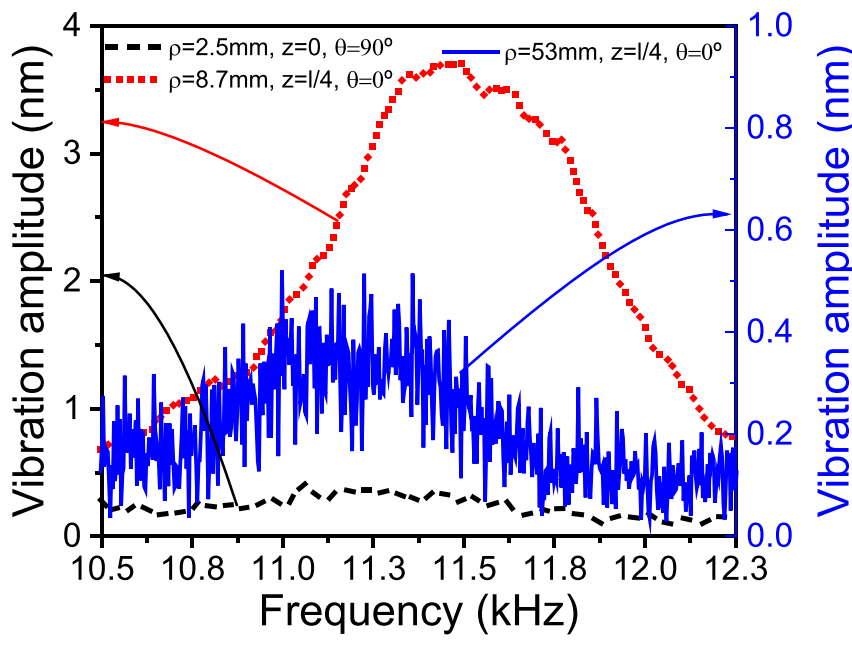

FIG. 4. Frequency response of the microcantilever out-of-plane vibration excited by the dipole antenna in three different conditions: the microcantilever antenna is placed at $\mathrm{z}=1 / 4$ with no tilt $\left(\theta=0^{\circ}\right)$ close (red dotted curve) and far (blue solid curve) from the dipole emitter. The microcantilever antenna is placed $(z=0)$ and oriented $\left(\theta=90^{\circ}\right)$ to be aligned with a null of the electric field (black dashed curve).

distance of $\rho=53 \mathrm{~mm}$ (being $\theta=0^{\circ}$ ). At this distance, we can easily measure the electric field ${ }^{23}(1.2 \mathrm{kV} / \mathrm{m})$ and we can obtain a responsivity of $\Re=0.29 \mathrm{~nm} / \mathrm{kV} / \mathrm{m}$.

However, when considering the micromechanical antenna as a power transducer, useful in energy harvesting or wireless power transfer applications, other characteristics become more relevant. For instance, the effective area, $A_{\text {eff }}$ which accounts for the capacity of the antenna to capture the available electromagnetic radiated power, is defined by ${ }^{22}$

$$
A_{\text {eff }}=\frac{P_{\text {mec }}}{\wp_{\text {rad }}} .
$$

Notice that we have adapted the classical definition of this parameter to our device by considering the mechanical power, $\mathrm{P}_{\mathrm{mec}}$, instead of the electrical power. Then, considering that the radiated power density, $\wp_{\mathrm{rad}}$, depends on the electric field intensity, E, as

$$
\wp_{\text {rad }}=\frac{E^{2}}{\eta}
$$

and the mechanical power associated with the vibration of the microcantilever at resonance is given by

$$
P_{m e c}=F v=\frac{q^{2} E^{2} \omega Q}{k},
$$

the effective area can be calculated as

$$
A_{\text {eff }}=\frac{q^{2} \omega Q \eta}{k}
$$

where we have set the exciting force in Eq. (5) as $\mathrm{F}=\mathrm{qxE}$, the amplitude of the vibration velocity at resonance in Eq. (5) is $\mathrm{v}=\omega \mathrm{xA}_{\mathrm{z}}$ and the vibration amplitude at resonance is $\mathrm{A}_{\mathrm{z}}=\mathrm{QxF} / \mathrm{k}$, with $\omega$ being the angular resonant frequency.
Besides, from Eqs. (6) and (7), we can easily obtain the power conversion efficiency, which is an even more relevant parameter when it comes to the comparison of the performance of our micromechanical antenna with radio frequency rectennas, as the state of the art reference for power transducers in RF energy harvesting and power transfer applications,

$$
\eta \stackrel{\text { def }}{=} \frac{P_{m e c}}{P_{\text {rad }}}=\frac{P_{m e c}}{\wp_{\text {rad }} A_{m a}}=\frac{A_{e f f}}{A_{m a}} .
$$

Here, we have used that $A_{m a}=0.5 \times B \times L$ [see Fig. 1(a)] is the area of the triangular mechanical antenna.

Finally, from Eqs. (6) and (7) and using all the microcantilever parameters reported above, we can calculate a value for the power conversion efficiency of $\eta=4 \times 10^{-9} \%$, which is orders of magnitude below the $20 \%$ efficiencies measured in microwave rectennas. However, since the size has a strong impact on future miniaturized self-powered devices, we have considered the ratio $\mathrm{FOM}=\eta / \mathrm{A}$ (with A being the area of the transducer) as an ad hoc figure of merit (FOM) that evaluates the power conversion efficiency and the size of the transducer at the same time. In these terms, the microcantilever antenna, with $\mathrm{FOM}=0.216 \% \mathrm{~m}^{-2}$, is only 4 orders of magnitude below the microwave antennas ${ }^{2}$ with $\mathrm{FOM}=8 \times 10^{3} \% \mathrm{~m}^{-2}(\eta=20 \%$, $\mathrm{A}=25 \mathrm{~cm}^{2}$ ).

In summary, we have fabricated a mechanical antenna from a commercial microcantilever by implanting a permanent charge that interacts with an oscillating electric field. We have characterized its mechanical frequency response when excited with a dipole antenna around the frequency of its first out-of-plane vibration mode. A responsivity of $\Re=0.29 \mathrm{~nm} / \mathrm{kV}$ m has been experimentally obtained. The power conversion efficiency per unit area has been defined and calculated to be $\mathrm{FOM}=0.216 \% \mathrm{~m}^{-2}$. Sound improvements of two orders of magnitude in the quality factor $(\mathrm{Q}=2000)$, which can be achieved by vacuum packaging, and one order of magnitude in the implanted charge $(\mathrm{q}=100 \mathrm{fC})$ would give an enhancement of the mechanical antenna performance of four orders of magnitude, $\mathrm{FOM}=2.16 \times 10^{3} \% \mathrm{~m}^{-2}$, which is comparable to the RF rectenna state of the art.

\section{REFERENCES}

${ }^{1}$ W. C. Brown, IEEE Trans. Microwave Theory Tech. 32(9), 1230-1242 (1984).

${ }^{2}$ J. A. Hagerty, F. B. Helmbrecht, W. H. McCalpin, R. Zane, and Z. B. Popovic, IEEE Trans. Microwave Theory Tech. 52, 1014-1024 (2004).

${ }^{3}$ K. V. Seshagiri Rao, P. V. Nikitin, and S. F. Lam, IEEE Trans. Antennas Propag. 53(12), 3870-3876 (2005)

${ }^{4}$ R. L. Bailey, J. Eng. Power 94(2), 73-77 (1972).

${ }^{5}$ G. A. E. Vandenbosch and Z. Ma, Nano Energy 1, 494-502 (2012).

${ }^{6}$ S. Joshi and G. Moddel, Appl. Phys. Lett. 102, 083901 (2013).

${ }^{7}$ A. Sharma, V. Singh, T. L. Bougher, and B. A. Cola, Nat. Nanotechnol. 10, 1027-1032 (2015).

${ }^{8}$ E. C. Anderson, T. L. Bougher, and B. A. Cola, Adv. Electron. Mater. 4, 1700446 (2018).

${ }^{9}$ J. Alda, J. M. Rico-García, J. M. López-Alonso, and G. Boreman, Nanotechnology 16, S230-S234 (2005).

${ }^{10}$ M. Bagmanci, M. Karaaslan, E. Unal, O. Akgol, M. Bakır, and C. Sabah, Int. J. Mod. Phys., B 33(8), 1950056 (2019).

${ }^{11}$ H. Wang, V. P. Sivan, A. Mitchell, G. Rosengarten, P. Phelan, and L. Wang, Sol. Energy Mater. Sol. Cells 137, 235-242 (2015).

${ }^{12}$ Y. Li, E. Baker, T. Reissman, C. Sun, and W. K. Liu, Appl. Phys. Lett. 111(25), 251903 (2017). 
${ }^{13}$ S. Qi, M. Oudich, Y. Li, and B. Assouar, Appl. Phys. Lett. 108(26), 263501 (2016).

${ }^{14}$ H. T. Zhong, X. X. Yang, X. T. Song, Z. Y. Guo, and F. Yu, Appl. Phys. Lett. 111(21), 213902 (2017).

${ }^{15}$ H. T. Zhong, X. X. Yang, C. Tan, and K. Yu, Appl. Phys. Lett. 109(25), 253904 (2016).

${ }^{16} \mathrm{G}$. Xu, H. Zhang, M. Xie, and Y. Jin, AIP Adv. 7(10), 105322 (2017).

${ }^{17}$ K. Jensen, J. Weldon, H. Garcia, and A. Zettl, Nano Lett. 7(11), 3508-3511 (2007).
${ }^{18}$ M. López-Suárez, J. Agustí, F. Torres, R. Rurali, and G. Abadal, Appl. Phys. Lett. 102, 153901 (2013).

${ }^{19}$ J. Wibbeler, G. Pfeifer, and M. Hietschold, Sens. Actuators, A 71, 74 (1998).

20 J. A. Malecki, Phys. Rev. B 59, 9954 (1999).

${ }^{21}$ NanoWorld ${ }^{\text {TM }}$ Pirex Nitride AFM probe: PNP-TR-TL.

${ }^{22}$ C. A. Balanis, Antenna Theory: Analysis and Design (Willey Interscience, 2003).

${ }^{23}$ Spectran NF-5020. 